“Transfer” XV: 1-2 (2020), pp. 351-370. ISSN: 1886-55

\title{
TRADUCCIONES ESPAÑOLAS DE LA OBRA DE ANNA DE NOAILLES.
}

Francisco Lafarga (ORCID 0000-0003-0847-5011) Universitat de Barcelona

Anna, condesa Mathieu de Noailles (1877-1933) según la nomenclatura oficial de la época en Francia -poco respetuosa de la identidad de la mujer- fue uno de los personajes más relevantes de la escena literaria y social del primer tercio del siglo XX. Aunque algún maldiciente afirmó que solo tenía de francesa el apellido de su marido, lo cierto es que esta greco-rumana de origen se sintió, desde su nacimiento parisiense, profundamente francesa y tuvo un dominio como pocos de la lengua francesa. $\mathrm{Su}$ salón fue lugar de encuentro de numerosos literatos y artistas del momento, con algunos de los cuales tuvo especial relación, de signo variado (Maurice Barrès, Marcel Proust). Su constante presencia en los actos sociales del grand monde no impidió su activa participación en distintas iniciativas en defensa de las libertades. Por otra parte, su nombre estuvo asociado al debate sobre la presencia de la mujer en la Académie française, que vetó una y otra vez el ingreso de escritoras; llegó a ser académica, aunque en Bélgica (Académie royale de langue et littérature françaises). ${ }^{1}$ Fundó, con otras escritoras, el premio Femina, y presidió el primer jurado del mismo (1904), que se reunió en su propia casa. ${ }^{2}$

\footnotetext{
Este trabajo se ha realizado en el marco del proyecto de investigación Portal digital de Historia de la Traducción en España, PGC2018-095447-B-I00 (MCIU/AEI/FEDER, UE).

${ }^{1}$ A su muerte, su sillón fue ocupado por otra mujer, Colette, quien dedicó a su amiga un sentido discurso; a Colette le siguió, en el mismo sillón, otro amigo de Anna de Noailles, Jean Cocteau.

${ }^{2} \mathrm{Su}$ apasionante figura y su rica trayectoria vital, así como su obra, han sido objeto de numerosos estudios. Ya en vida aparecieron algunos, debidos a G.-A. Masson (1922), R. Benjamin (1928) y J. Larnac (1931), mientras que críticos como L. Blum (1906) o Ch. Du Bos (1922) le dedicaron su atención. Tras un largo periodo de olvido, en el que solo figura el libro de su amiga la duquesa de La Rochefoucauld (1956), el interés de la crítica renace en los años 1980 con las biografías de Cl. Mignot-Ogliastri (1986) y F. Broche (1989), a las que hay que añadir la reciente de F.
} 
“Transfer” XV: 1-2 (2020), pp. 351-370. ISSN: 1886-55

\section{Recepción}

La presencia de Anna de Noailles en España, según se desprende del análisis de la prensa del primer tercio del siglo XIX, fue sostenida y variada, pues sus actividades literarias, mundanas y sociales dieron pie a múltiples noticias y comentarios. Contribuyeron asimismo a aumentar la presencia unos anunciados viajes a Madrid y a Barcelona, que nunca se realizaron, y el retrato que hizo de ella Ignacio Zuloaga en $1913 .^{3}$

Obviamente, estos motivos hicieron que su presencia en España, o el conocimiento que de ella podía tener el público lector español, fuera superior al de sus contemporáneas Gyp, Séverine, Rachilde, Myriam Harry, Marcelle Tynaire, Lucie Delarue-Mardrus o Gérard d'Houville. Tal vez Colette fue la única que suscitó parecido interés.

Aunque, como se verá en el siguiente apartado, las traducciones de Anna de Noailles fueron parciales y poco numerosas, su impacto literario puede medirse también por numerosos artículos de prensa que van más allá de la noticia, aunque en ocasiones se originen en ella (por ejemplo, los surgidos a raíz de su muerte). Este no es el lugar para referirme con la debida atención a estos

Martinez (2018). También se constata una recuperación de su obra con varias reediciones, entre las que destaca la de su poesía completa en tres volúmenes, al mismo tiempo que han aparecido varios estudios de envergadura gracias a C. Perry (2003), R. M. Verona (2011) y M.-L. Allard (2013). Conviene asimismo mencionar dos sitios en la red: la página Anna de Noailles, creada por Catherine Perry en 2009 (disponible en: <<http://www.annadenoailles.org/>>) y un blog, de autor desconocido, activo entre 2010 y 2016, con casi ochocientos archivos (disponible en: $<<$ http://comtessedenoailles.blogspot.com/>>; última consulta: 15.09.2019). En ámbito español, tras el artículo de Ortega y Gasset (1923) conviene mencionar el librito de M. Barbeito (1962) y el estudio de A. Piquer (1996), además de las páginas que le dedica C. Pujante (2014).

${ }^{3}$ La obra, que es propiedad del Museo de Bellas Artes de Bilbao, produjo la reacción literaria de Rafael Sánchez Mazas con sus "Siete sonetos ante el retrato de la condesa de Noailles", publicados en 1917 en la revista Hermes ( $n^{\circ}$ 8, 521-522). Como curiosidad, puede añadirse que formó parte de la serie de ocho sellos dedicada a Zuloaga por la FNMT en 1971. Véase, para la relación entre Zuloaga y su modelo, Rementería (2001). 
“Transfer” XV: 1-2 (2020), pp. 351-370. ISSN: 1886-55

artículos, y me limitaré a recordar los nombres de sus autores, periodistas y escritores, unos desde España y otros desde Paris, donde ejercían de corresponsales de distintos periódicos de la época. Así, pueden hallarse en la prensa diaria y en revistas artículos específicos sobre Anna de Noailles por Melchor de Almagro, Luis Araujo Costa, Carlos de Batlle, Cristóbal de Castro, Carmen Conde, Concepción Gimeno de Flaquer, Enrique Gómez Carrillo, José $\mathrm{M}^{\mathrm{a}}$ López-Picó, Elisabeth Mulder, Margarita Nelken, Manuel Núñez de Arenas, José Ortega y Gasset, Matilde Ras, Félix Ros o Carles Soldevila. Y nuestra autora tiene también un lugar destacado en colaboraciones periodísticas de autores como Azorín, Colombine, Alberto Insúa y Mauricio López Roberts.

\section{Traducciones}

Utilizando distintas fuentes he podido reunir las traducciones de obras de Anna de Noailles, que aparecen en apéndice. Pueden hacerse algunas consideraciones generales antes de dar paso a la descripción de las distintas versiones.

En primer lugar, la cronología de las traducciones tiene un punto de inflexión, a mi entender, en el momento del fallecimiento de la autora (30 de abril de 1933), con lo cual podría distinguirse entre traducciones publicadas (o realizadas) en vida de Anna de Noailles y tras su muerte.

Otra consideración tiene que ver con el medio de difusión de las traducciones. Las primeras se publicaron ya sea en la prensa, ya en antologías. De hecho, hasta 1931 no apareció una traducción en forma de libro (El rostro maravillado, obra de Tomás Borrás), y hubo que esperar a 1948 para hallar una nueva versión en volumen, la de Una carta de las que no se envían por Rosa Chacel, y de ahí otro paréntesis hasta 2011, año de la publicación de un volumen de poesías escogidas (titulado Las pasiones y las tumbas), obra de Mireia Alonso Ribeiro. ${ }^{4}$

\footnotetext{
${ }^{4}$ Aun cuando las he incluido en la relación de traducciones del apéndice, no puedo tomar en consideración en este recorrido histórico dos versiones que no me ha
} 
“Transfer” XV: 1-2 (2020), pp. 351-370. ISSN: 1886-55

Otra vía más actual es la de la difusión en línea, poco explotada en el caso que nos ocupa, pero de la que hay algún ejemplo, que se indicará en su lugar.

La más antigua traducción publicada que he podido localizar es la del poema "Silencio en verano", que apareció sin el nombre del traductor en la revista Por esos mundos en julio de 1908. Con todo, debe atribuirse a Eduardo Marquina, ${ }^{5}$ ya que el texto es, salvo una ligera variante, idéntico al que, con su nombre, se incluyó en La poesía francesa moderna de Enrique Díez-Canedo y Fernando Fortún, aparecida en $1913 .^{6}$

Esta conocida antología presenta cuatro composiciones de Anna de Noailles, la ya mencionada en traducción de Marquina, otra debida a Díez-Canedo, y dos obra de F. Fortún; también, como es usual en ella, incluye una breve nota biográfica de la autora. La riqueza y variedad de sus contenidos, así como el buen hacer de sus colaboradores labraron una merecida fama a esta antología, referente en su época, que contribuyó a la difusión de la figura y la obra de Anna de Noailles.

Parte de uno de los poemas presentes en La poesía francesa moderna, el "Frente a España" traducido por F. Fortún, fue reproducido (aunque solo con las iniciales del traductor) en julio de 1915 como ilustración en un artículo no firmado de la revista Iberia dedicado a "La poesía francesa de España", que contiene asimismo un fragmento del poema "Les Pyrénées" de Edmond Rostand, traducido por el poeta Manuel de Sandoval.

Cierta presencia tuvo también la poesía de Anna de Noailles en las antologías preparadas por Fernando Maristany, desde Poesías excelsas (breves) de los grandes poetas, de 1914, hasta la Antología general de poetas líricos franceses de 1921, pasando por el Florilegio del año anterior. Llama la atención que Maristany no

sido posible consultar (de El libro de mi vida y El rostro maravillado, publicadas en Buenos Aires en 1932 y 1945, respectivamente), que mencionaré en el lugar correspondiente.

${ }_{5}^{5}$ Sobre la labor como traductor de E. Marquina puede verse Marco García (2001), Léglise (2014), Hambrook (2016) y García Garrosa (2018).

${ }^{6}$ Han estudiado distintos aspectos de esta antología Pym (1995), Gallego Roca (1996), Lama (1999-2000), Gómez Bedate (2001), Giné (2017) y Lama (2018). 
“Transfer” XV: 1-2 (2020), pp. 351-370. ISSN: 1886-55

incluyera ningún poema en Las cien mejores poesías (líricas) de la lengua francesa, una de las antologías que alcanzó mayor difusión. En cualquier caso, Anna de Noailles está presente con tres poemas en el conjunto de las traducciones de Maristany, y es una de las escasas mujeres con obra traducida. ${ }^{7}$

La mayor parte de los textos traducidos, sobre todo en forma fragmentaria y con publicación en antologías o prensa, son poemas. Son los que han aparecido hasta este punto en el recorrido cronológico que he juzgado más conveniente para la descripción de las versiones y los que mayoritariamente aparecerán en los párrafos que siguen.

Hay, con todo, alguna excepción, y la más antigua es un texto sobre la moda, con no pocas -y esperables- disquisiciones literarias que Anna de Noailles redactó en forma de carta dirigida a George Barbier y que debía incorporarse como prólogo o presentación del volumen de 1922 de Falbalas et fanfreluches. Almanach des modes présentes, passées et futures. ${ }^{8}$ Con todo, apareció previamente en el periódico Le Gaulois (el 29 de diciembre de 1921), de donde lo tomó el diario madrileño La Época para su suplemento dominical del 21 de enero siguiente.

Una nueva aparición de la poesía se produjo algunos años más tarde (probablemente en 1925) ${ }^{9}$ en el monumental Libro de los poetas del presbítero Juan Díaz Quevedo: se trata del poema que titula "El sol en el postigo", que no es otro que "Silence en été" de Les éblouissements, precisamente el que Marquina tenía ya traducido en 1908, y constituye la primera aparición de un texto de Anna de Noailles en la bibliografía española. ${ }^{10}$

\footnotetext{
7 Son varios los estudios sobre la figura de traductor y antólogo de F. Maristany, entre ellos: Pym (1995), Gallego Roca (1996), Sáez Delgado (2007-2011), Camps (2017), Zarandona (2017), Gasó (2018) y Lafarga (en prensa).

${ }^{8}$ Dibujante e ilustrador, Barbier sacó entre 1922 y 1926 cinco volúmenes de Falbalas et fanfreluches, que contenían dibujos de indumentaria de corte modernista e incluían un texto de alguna celebridad, como las escritoras Colette, Gérard d'Houville o Renée de Brimont (además de la condesa de Noailles) o la actriz y cantante Cécile Sorel.

${ }^{9}$ Hay una reseña en $A B C$ de 22.01.1926.

${ }^{10}$ Díaz Quevedo (1884-1951) fue un sacerdote canario. Además de El libro de los poetas (1925) dio Prosas de un ensayista (1940).
} 
“Transfer” XV: 1-2 (2020), pp. 351-370. ISSN: 1886-55

Tras este poema hubo que esperar varios años hasta la aparición de la primera traducción en forma de libro, la de El rostro maravillado, realizada por Tomás Borrás ${ }^{11}$ y publicada en 1931 por la editorial Estrella en la difundida colección "Biblioteca Estrella" de libros en pequeño formato, bellamente editados. Aunque en la traducción aparece calificada de "novela", se trata en realidad de un diario, a lo largo de un año, en el que una joven monja relata su vida en el convento, sus anhelos, sus dudas y -en particular- el desasosiego que le produce el sentimiento amoroso hacia un joven pretendiente. De esta traducción apareció años más tarde, aunque sin fecha, una edición fraudulenta, obra de cierto J. V., aunque el cotejo señala que es idéntica a la de Borrás. Se dice editada en París, en la colección "Clair de Lune", aunque consta impresa en México. ${ }^{12}$

Ya en el límite de la primera época a la que aludía al inicio, se sitúan dos versiones al catalán realizadas entre 1933 y 1934 por Màrius Torres, que -como el resto de su obra- no se publicaron en su momento, y aun han debido esperar más tiempo que sus poemas originales, ya que no han aparecido hasta 2010, coincidiendo con el centenario de su nacimiento. Se trata de dos

\footnotetext{
T. Borrás (1891-1976) fue un afamado periodista y prolífico autor de relatos breves, novelas y piezas teatrales, además de traductor. Vertió sobre todo obras teatrales de notables autores franceses: El avaro y El casamiento a la fuerza (1919) de Molière, Juego de amor y de azar y El legado (1920) de Marivaux, Lorenzaccio (1921) de Musset o La escuela de los indiferentes (1921) de Giraudoux. Del teatro inglés, Volpone (1953) de Ben Jonson y del italiano, Enrique IV (1940) y La vida que te di (1944) de Pirandello. Dio también varias versiones de piezas húngaras, traducidas en colaboración, como ¡Atrévete, Susana! (1929), ¡Casáte con mi mujer! (1930) y Suspenso en amor (1941) de Ladislas Fodor, y Olimpia (1930) de Ferenc Molnár; así como un arreglo para el teatro de la novela Fabiola del cardenal N. Wiseman (1930, con V. de Pedro). Vertió asimismo varios relatos de dos contemporáneos de la condesa de Noailles: La felicidad de Ginette (1921) de Gyp (Sibylle de Riquetti de Mirabeau) y La pecadora (1930) de Henri de Régnier.

${ }_{12}$ No me ha sido posible acceder a otra versión de la obra, por Poldy de Bird, publicada en Buenos Aires por la editorial Claridad en 1945 (col. "La Nave"). Tampoco he podido consultar la única traducción publicada en español de Le livre de ma vie, realizada por Pedro Labrousse y publicada asimismo en Buenos Aires por Ediciones Cóndor en 1932.
} 
“Transfer” XV: 1-2 (2020), pp. 351-370. ISSN: 1886-55

poemas del volumen Les forces éternelles, "A mon fils" y "Destin", que pueden leerse en dos ediciones aparecidas el mismo año. ${ }^{13}$

La segunda etapa de las traducciones se inicia, de hecho, varios años después de la muerte de la autora $y$, aunque obra de una española, se sitúa a miles de kilómetros de distancia. Como es sabido, en la larga época del exilio Rosa Chacel halló en la traducción una fuente de ingresos. ${ }^{14}$ Una de las obras que tradujo en esta época y que se publicó, como la mayoría, en Argentina, fue la nouvelle de Anna de Noailles Parmi les lettres qu'on n'envoie pas, incluida en el volumen Les innocentes.

Aparecida con fecha de 1948, se trata de una edición de gran formato $(37 \mathrm{~cm})$, aunque muy breve (23 páginas), de tirada corta (500 ejemplares numerados), adornada con dos ilustraciones de Juan Batlle Planas ${ }^{15}$ y el retrato de la autora por Raúl Soldi. Además, el signo de la colección, una perdiz, que aparece en la portada, es obra de Norah Borges, según consta en el colofón. Un conjunto, pues, de elementos para un producto de gran belleza y refinamiento.

Esta traducción parece una isla o un faro, en la bibliografía española de Anna de Noailles: es lo único que consta entre 1931, año de El rostro maravillado de T. Borrás (o, si se quiere, 1932, año de El libro de mi vida de P. Labrousse, que no he visto) y 1985, momento en el que se publicó una antología de poesía francesa en lengua catalana, que contenía los poemas "Le temps de vivre" (de

${ }^{13}$ Precisamente una de ellas contiene un estudio sobre Torres como traductor (véase Ballart y Julià 2010).

${ }^{14}$ Varias traducciones de R. Chacel, además de la de A. de Noailles, aparecieron en Argentina: La peste de Camus (1948); Antígona y Reinaldo y Armida de Cocteau (1952); Reunión de familia de T. S. Eliot (1953); Animales desnaturalizados de Vercors (1953); Edén Término. El retamal. Cornelius de J. B. Priestley (1957), y Libertad o muerte de Kazantzakis (1957). En La Habana se publicó Herodías de Mallarmé (1957). Ya en España aparecieron Seis tragedias de Racine (1983) y el poemario Museo de cámara de Walmir Ayala (1986). Existen varios estudios sobre distintos aspectos de la labor traductora de R. Chacel, en particular de González Ródenas (2001), Lafarga (2001), Gómez Bedate (2006) y Behiels (2018).

${ }^{15}$ Batlle Planas (1911-1966), pintor de origen catalán, aunque vivió desde niño en Argentina; en el texto aparece como Battle. Por su parte, Soldi (1905-1994) es uno de los más renombrados artistas argentinos de su época. Norah Borges (19011998), hermana de Jorge Luis, fue una célebre pintora vanguardista. 
“Transfer” XV: 1-2 (2020), pp. 351-370. ISSN: 1886-55

Le cour innombrable) y "Offrande" (de Les éblouissements), ambos traducidos por Joan Peña. La antología pertenece a una prestigiosa colección de traducciones de literatura universal.

De anecdótica puede tildarse la inclusión en el $A B C$ (Sevilla) de 18 de agosto de 1995 de cuatro versos del poema "Il fera longtemps clair ce soir..." (de Le cour innombrable) que aparecen en la estela dedicada a Anna de Noailles en el llamado Jardin des Poètes de París, cerca del bosque de Boulogne, en francés y con traducción castellana de F. Arrabal, Fernando, sin duda, a quien se deben igualmente unos comentarios provocadores sobre la condesa.

Por su parte, la escritora Mireia Alonso Ribeiro ha contribuido a la difusión de la poesía de la condesa de Noailles en dos momentos y por dos vías distintas, la prensa y la antología. ${ }^{16}$ Así, en la revista literaria Fábula publicó en 2007 cuatro poemas procedentes de Les vivants et les morts, con texto bilingüe. Los textos van precedidos de una presentación de la autora y de su obra, y acompañados de varias imágenes de Anna de Noailles, entre ellas la que le dedicó J. Cocteau al poco tiempo de su muerte, con esta inscripción: "Je ferme les yeux. J'essaye, Anna, de revoir votre sourire". Llama la atención, sin embargo, que la autora utilizara como título de su artículo el de uno de los poemarios de la condesa (Le coeur innombrable), cuando los cuatro poemas que tradujo proceden de Les vivants et les morts.

Mireia Alonso adoptó un procedimiento parecido en el volumen antológico que publicó en 2011, al que dio el título de Las pasiones y la tumbas, que recoge la denominación de dos apartados de Les vivants et les morts. La insistencia en estos dos aspectos en la introducción (pp. 9-18), por una parte la pasión amorosa, el deseo, y por otra la muerte, el abismo, la pérdida, justifican la selección de los veinticinco poemas de que consta el volumen, extraídos de nueve libros de Anna de Noailles. Esta

\footnotetext{
${ }^{16}$ Por la nota que se ha insertado en el volumen Las pasiones y las tumbas, mencionado más abajo, sabemos que la autora ha cursado estudios de filología y ha sido profesora de español en varios centros. Ha escrito para el teatro y ha publicado varios libros de poesía. Ha traducido la obra de Arthur Cravan (2012) y de Anna de Noailles también El libro de mi vida (que no consta impreso).
} 
“Transfer” XV: 1-2 (2020), pp. 351-370. ISSN: 1886-55

multiplicidad de fuentes demuestra la singular presencia de las dos líneas en el universo poético de la autora; con todo, llama la atención la disposición de los textos contraria a la cronología, en ocasiones con saltos de más de veinte años, hacia adelante y hacia atrás. La traductora no lo justifica y no hay ninguna razón aparente que obligue a ello, dentro de la temática de cada bloque. Por otra parte, aunque en un apéndice (pp. 61-75) ofrece los textos originales, no se especifica de qué poemario han sido sacados, lo cual es -a mi entender- una grave falta de rigor filológico...

En época más reciente (2014), la publicación de la traducción del volumen Cartas a la condesa de Noailles, que forma parte de la correspondencia de Marcel Proust, obra de Alfonso GarcíaSampedro y Caroline Le Lanchon, ha aportado nuevas versiones de nuestra autora, tanto las propias notas como varios textos de acompañamiento, que ya figuraban en la edición francesa de la correspondencia.

Y para cerrar este itinerario, volvemos a la poesía, aunque en esta ocasión en formato digital, que es el adoptado por Julio Pollino Tamayo para difundir su traducción de un poemario completo (único ejemplo existente): El honor de sufrir. ${ }^{17}$

La relación de las traducciones existentes, que me temo será incompleta, puede ampliarse con la mención de traducciones que no fueron, o que no llegaron a ser. Así, se sabe de la existencia de una versión de poemas de Anna de Noailles llevada a cabo por el poeta catalán Ricardo de Mir Massana. ${ }^{18}$ Desconozco cual fue el alcance de esa traducción, de la que la prensa de Barcelona se hizo eco en varias ocasiones. Tanto La Vanguardia (en la edición de 18.03.1934) como El Diluvio (el 17.04.1934) publicaron una gacetilla sobre un banquete de homenaje ofrecido por sus amigos y admiradores, en especial "por sus recientes traducciones de obras de la condesa de Noailles, tan bien recibidas en los medios literarios". Se comenta incluso que el traductor había obtenido la auto-

\footnotetext{
17 No he conseguido datos biográficos del traductor: en la red hay muchas publicaciones suyas en línea, la mayoría sobre cine, aunque también alguna traducción, como la de El amor La poesía de P. Éluard.

${ }^{18}$ De este autor solo puedo decir que publicó en la década de 1920 tres libros de poesías: Horas líricas, Los luminares y Las plumas del yelmo.
} 
“Transfer” XV: 1-2 (2020), pp. 351-370. ISSN: 1886-55

rización del hijo de la autora, Anne-Jules de Noailles, pues su madre había fallecido un año antes (La Vanguardia, 04.05.1934). En cualquier caso, el fallecimiento del traductor en diciembre del mismo año le impidió llevar a buen puerto la empresa: "La muerte le ha sorprendido cuando estaba acabando de dar cima a la traducción de un manojo de poemas de la poetisa condesa de Noailles, tarea en la que ponía todo su entusiasmo y toda su ilusión" (El Diluvio, 13.12.1934, p. 7).

Otra traducción que no llegó a ver la luz fue la de María Barbeito $^{19}$ de El libro de mi vida, aunque en alguna fuente aparece como publicada, incluso con pie de imprenta y fecha (Madrid, Imprenta Juan Bravo 3, 1962). Parece que efectivamente se llevó a cabo, aunque lo único que se publicó (con el mismo título y pie de imprenta) fue el prólogo (véase Castro 2011: 122).

Y, como ya he mencionado más arriba, habría que añadir a esta lista la traducción de la misma obra, atribuida a Mireia Alonso en una nota de su versión de poesías Las pasiones y las tumbas, y que no consta como publicada.

\section{Conclusión}

A lo largo de este trabajo ha quedado, creo, demostrada la continuada presencia de traducciones de obras de Anna de Noailles en lengua española, en una larga historia que se inicia en 1908 y llega hasta el presente. Es justo señalar, con todo, el carácter fragmentario de dicha presencia, con publicaciones dispersas y en raras ocasiones con traducción de la obra completa, ya sea poemarios, ya obras en prosa. Al mismo tiempo, se aprecia la recuperación de la autora en los últimos años en el ámbito de la traducción, en consonancia con la nueva presencia de su obra en el mercado editorial francés. Obviamente, las traducciones no manifiestan la riqueza y la variedad de su escritura: se ha privilegiado la poesía,

\footnotetext{
${ }^{19}$ M. Barbeito (1880-1970) fue pedagoga y escritora, miembro de la Real Academia Gallega. Apartada de la docencia tras la Guerra Civil, se dedicó a la escritura y a la traducción, aunque la mayor parte de su producción ha quedado inédita.
} 
“Transfer” XV: 1-2 (2020), pp. 351-370. ISSN: 1886-55

que fue lo más alabado de su producción en vida, aunque han quedado en el olvido (también en Francia) la mayor parte de sus prosas, fundamentalmente poéticas.

\section{Apéndice: Relación de las traducciones}

1908

"Silencio en verano", sin nombre de traductor, en Por esos mundos XVII (julio de 1908), 78 < "Silence en été" (de Les éblouissements, 1907) [idéntica, salvo una variante, a la de E. Marquina en la antología de Díez-Canedo y Fortún de 1913]

1913

"Il fera longtemps clair ce soir..." ["Será largo el crepúsculo..."], trad. de Enrique Díez-Canedo < "Il fera longtemps clair ce soir..." (de Le cœur innombrable, 1901); "Frente a España", trad. de Fernando Fortún < "En face de l'Espagne" (de Les éblouissements, 1907); "Silencio en verano", trad. de Eduardo Marquina < "Silence en été" (de Les éblouissements, 1907); "Tarde vasca", trad. de F. Fortún < "Soir basque" (de Les éblouissements, 1907) en: Enrique Díez-Canedo \& Fernando Fortún. (eds.). (1913). La poesía francesa moderna. Madrid: Renacimiento, pp. 279-282.

1914-1921

"Elevación", trad. de Fernando Maristany < "Élévation" (de Les vivants et les morts, 1913) en: Florilegio. Las mejores poesías líricas griegas, latinas, italianas, portuguesas, francesas, inglesas y alemanas. Barcelona: Cervantes, 1920, pp. 473-474, y en: Antología general de poetas líricos franceses (1391-1921). Barcelona: Cervantes, 1921, pp. 287-288.

"Escribo a fin que cuando mi vida...", trad. de Fernando Maristany < "J'écris pour que le jour..." (de L'ombre des jours, 1902) en: Poesías excelsas (breves) de los grandes poetas. Barcelona: A. López, 1914, p. 171, y Antología general, p. 287. 
“Transfer” XV: 1-2 (2020), pp. 351-370. ISSN: 1886-55

"La imagen", trad. de Fernando Maristany < "L'image" (de Le cœur innombrable, 1901) en: Florilegio, pp. $472-473$ y en: Antología general, pp. 285-286.

1915

"Frente a España" (fragmentos), trad. de Fernando Fortún en: "La poesía francesa a España", Iberia I (03.07.1915), 13: 4 < "En face de l’Espagne" (de Les éblouissements, 1907).

1922

"Carta sobre la moda", sin nombre de traductor en: La Época (suplemento dominical) 21.01.1922, p. 2 < "Lettre sur la mode" en: Le Gaulois (29.12.1921), p. 1, luego en: George Barbier. (1922). Falbalas et fanfreluches. Almanach des modes présentes, passées et futures pour 1922. París: Meynial.

¿1925?

"El sol en el postigo" en: Juan Díaz Quevedo. (s. a. ¿1925?). El libro de los poetas. Antología universal del arte de la lectura. Madrid: Fernando Fe,, p. 233 < "Silence en été" (de Les éblouissements, 1907).

1931

El rostro maravillado (Novela). Traducción de Tomás Borrás, Madrid, Estrella, 1931 (“Biblioteca Estrella”), 174 pp. < Le visage émerveillé (1904); hay una reed. de París: Colección Clair de Lune, s. a., atribuida a J. V., con el texto idéntico.

1932

El libro de mi vida. Traducción de Pedro Labrousse. Buenos Aires: Ediciones Cóndor, 1932, 185 pp. ("Las grandes biografías contemporáneas", 6).

$1933-1934 / 2010$

“Al meu fill” y "Destí" en: Màrius Torres (2010). Versions de poesia europea. Ed. y estudio de Pere Ballart y Jordi Julià. Lleida: Pagès, 
“Transfer” XV: 1-2 (2020), pp. 351-370. ISSN: 1886-55

pp. 150-153 < “À mon fils” y "Destin" (de Les forces éternelles, 1920).

$1933-1934 / 2010$

“Al meu fill” y "Destí" en: Màrius Torres. (2010). Màrius Torres tradueix. Ed. de Miquel Àngel Aguado y Txema Martínez. Lleida: Alfazeta, pp. 73-76 < “À mon fils" y "Destin" (de Les forces éternelles, 1920).

1945

El rostro maravillado. Traducción de Poldy de Bird. Buenos Aires: Claridad, 1945, 151 pp. ("La Nave").

1948

Una carta de las que no se envían. Con dos ilustraciones de Battle Planas y un retrato de la autora por Raúl Soldi. Traducción de Rosa Chacel. Buenos Aires: Ediciones Arturo Jacinto Álvarez, 1948 (“Colección La Perdiz”), 22 pp. < “Parmi les lettres qu'on n'envoie pas” (en: Les innocentes, 1923, pp. 62-83).

1985

"El temps de viure" y "Ofrena", trad. de Joan Peña en: Alain Verjat (ed.). (1985). Poesia francesa. Antologia del segle XIII al XIX. Barcelona: Edicions 62, pp. 434-436 ("Millors Obres de la Literatura Universal") < "Le temps de vivre" (de Le cour innombrable, 1901) y "Offrande" (de Les éblouissements, 1907).

1995

"Il fera longtemps clair ce soir..." (cuatro versos) por F.[ernando] Arrabal en: "El jardín de los poetas muertos", ABC Cultura 18.08.1995, p. 37 < "Il fera longtemps clair ce soir..." (de Le cœur innombrable, 1901).

2007

"Amarte, y cuando el tímido día...", "No puedo comprender...", "Ternura" y "No me alegro de nada..." en: Mireia Alonso Ribeiro. (2007). "Un corazón innombrable: Anna de Noailles (1876-1933)", 
“Transfer” XV: 1-2 (2020), pp. 351-370. ISSN: 1886-55

Fábula. Revista literaria 23 (2007): 74-84 < “T’aimer et quand le jour timide...", "Je ne puis pas comprendre...", “Tendresse” y "Je ne me réjouis de rien..." (de Les vivants et les morts, 1913).

2011

Las pasiones y las tumbas. (Edición bilingüe). Prólogo y traducción de Mireia Alonso Ribeiro. Madrid: Torremozas, 2011, 77 pp. (“Colección Torremozas", 252).

Las pasiones: "No puedo comprender...", 21-22 < "Je ne puis pas comprendre..." (de Les vivants et les morts, 1913); "Aguarda un poco aún...", 23-25 < "Attends encore un peu..." (de Les forces éternelles, 1920); "CXLII", 26 < "CXLII" (de Poème de l'amour, 1924); "Nuestro amor", 27-28 < "Notre amour" (de Poèmes d'enfance, 1928); "Desgarro", 29-31 < "Déchirement" (de Les éblouissements, 1907); "La Naturaleza y el Hombre", 32-34 < "La Nature et l'Homme" (de Le coeur innombrable, 1901); "La nostalgia", 35-36 < "La nostalgie" (de Les éblouissements, 1907); "Habíamos esperado...", 37 < "Nous avons attendu..." (de Les forces éternelles, 1920); "La adolescencia", 38 < "L'adolescence" (de L'ombre des jours, 1902); "XXIV", 39 < "XXIV" (de Poème de l'amour, 1924); "Tarde de verano", 40 < "Soir d'été" (de Le cour innombrable, 1901); "Después de los momentos...", 41 < “C'est après les moments..." (de Les forces éternelles, 1920); "CXLVIII", 42 < "CXLVIII" (de Poème de l'amour, 1924).

Las tumbas: "El abismo", 45-46 < "L'abîme" (de Les vivants et les morts, 1913); "Yo no quiero saber...", 47 < "Je ne veux pas savoir... (de Les vivants et les morts, 1913); "Yo respiro y tú duermes...", 48 < "Je respire et tu dors..." (de Les vivants et les morts, 1913); "Vivía. Mi mirada, cual colonia...", 49-51 < "Je vivais. Mon regard, comme un peuple..." (de Les vivants et les morts, 1913); "Todo se escapa, el hombre...", 52 < "Tout nous fuit..." (de Les forces éternelles, 1920); "Mi espíritu ansioso...", 53 < "Mon esprit anxieux..." (de Les forces éternelles, 1920); "XXIX", 54 < "XXIX" (de L'honneur de souffrir, 1927); "XLV", 55 < "XLV" (de L'honneur de souffrir, 1927); "Misión", 56 < "Mission" (de Derniers vers, 1933); "Obsesión", 57 < "Obsession" (de Derniers vers, 1933); "Interrogación", 58 < "Interrogation" (de Les forces éternelles, 
“Transfer” XV: 1-2 (2020), pp. 351-370. ISSN: 1886-55

1920); "El desamparo", 59 < "La détresse" (de L'ombre des jours, 1902).

2014

"Retrato de Marcel Proust" (pp. 13-19), "Un recuerdo de Marcel Proust” (pp. 21-26) y "Cuando Marcel Proust nos dejó" (pp. 27-30) en: Marcel Proust. (2014). Cartas a la condesa de Noailles. Presentadas y anotadas por la condesa de Noailles. Traducción de Alfonso García-Sampedro y Caroline Le Lanchon. Sevilla: Metropolisiana Ediciones < "Portrait de Marcel Proust", "Un souvenir de Marcel Proust" y "Quand Marcel Proust nous eut quittés" en: Marcel Proust (1931). Lettres à la comtesse de Noailles, (vol. II de la Correspondance générale de M. Proust).

2018

El honor de sufrir. Traducción de Julio Pollino Tamayo, sitio web SlideShare, 2018.

$<<$ https://es.slideshare.net/JulioPollinoTamayo/el-honor-desufrir-19231927-anna-de-noailles-poemario>>

$<$ L'honneur de souffrir (1927).

\section{Referencias bibliográficas}

ALLARD, Marie-Lise. (2013). Anna de Noailles: entre prose et poésie. París: l'Harmattan.

BALLARD, Pere \& JULIÂ, Jordi. (2010). "Màrius Torres, traductor de poesia europea". En Torres, Màrius, Versions de poesia europea. Lleida: Pagès Editors, pp. 7-41.

BARBEITO, María. (1962). La condesa de Noailles. Madrid, s. i. (Imp. Juan Bravo 3).

BEHIELS, Lieve. (2018). "Rosa Chacel: novelista y traductora española exiliada”. Cadernos de Tradução 38(1): 47-64. Disponible en: 
“Transfer” XV: 1-2 (2020), pp. 351-370. ISSN: 1886-55

$<<$ https://periodicos.ufsc.br/index.php/traducao/article/vi ew/2175-7968.2018v38n1p47/35753>> [consultado el 15.09.2019].

BENJAMIN, René. (1928). Au soleil de la poésie. Sous l'œil en fleur de Madame de Noailles. París: Librairie des Champs-Élysées.

BLUM, Léon. (1906). En lisant. Réflexions critiques. París: P. Ollendorff.

BROCHE, François. (1989). Anna de Noailles. Un mystère en pleine lumière. París: Robert Laffont.

CAMPS, Assumpta. (2017). "Las traducciones de poesía italiana en el Florilegio de Fernando Maristany: ¿una antología de autor?". En: LAFARGA, Francisco. (ed.). La traducción fragmentaria: su lugar en antologías y revistas (1898-1936). Madrid: Guillermo Escolar, pp. 37-52.

CASTRO VÁZQUEZ, Olga. (2011). "Traductoras gallegas del siglo XX: reescribiendo la historia de la traducción desde el género y la nación". MonTI 3: 107-130 (Woman and Translation: Geographies, Voices and Identities).

DU BOS, Charles. (1922). Approximations. Première série. París: Plon.

GARCÍA GARROSA, M. ${ }^{\mathrm{a}}$ Jesús. (2018). "Eduardo Marquina, ¿traductor a su pesar?”. En: LAFARGA, Francisco. (ed.), Creación y traducción en España (1898-1936): protagonistas de una historia. Kassel: Reichenberger, pp. 109-125.

GASÓ, Nuria. (2018). "La estela de Fernando Maristany y Manuel de Montoliu en la traducción de tres poetas alemanes (Novalis, Rilke y Hölderlin) durante el siglo XX". 1611 12. Disponible en:<<http://www.traduccionliteraria.org/1611/art/gaso.ht $\mathrm{m}>>$ [consultado el 15.09.2019].

GINÉ, Marta. (2017). "La poesía francesa moderna de E. DíezCanedo y F. Fortún y sus originales, de Aloysius Bertrand a Paul Fort”. En: LAFARGA, Francisco. (ed.). La traducción fragmentaria: su lugar en antologías y revistas (1898-1936). Madrid: Guillermo Escolar, pp. 65-90.

GÓMEZ BEDATE, Pilar. (2001). "Nota sobre los temas simbolistas en La poesía francesa moderna de Enrique Díez-Canedo y 
“Transfer” XV: 1-2 (2020), pp. 351-370. ISSN: 1886-55

Fernando Fortún". En: PEGENAUTE, Luis. (ed.). La traducción en la Edad de Plata. Barcelona: PPU, pp. 89-98. Disponible en:

$<<$ http://www.cervantesvirtual.com/obra/notas-sobre-lostemas-simbolistas-en-la-poesia-francesa-moderna-deenrique-diez-canedo-y-fernando-fortun/ $>>$ [consultado el 15.09.2019].

GÓMEZ BEDATE, Pilar. (2006). “A propósito de la traducción de Herodías por Rosa Chacel”. Insula 717: 18-20.

GÓNZALEZ RÓDENAS, Soledad. (2001). "Rosa Chacel: creación, traducción y crítica. A propósito de seis tragedias de Racine". En: LAFARGA, Francisco \& DOMÍNGUEZ, Antonio. (eds.). Los clásicos franceses en la España del siglo XX. Estudios de traducción y recepción. Barcelona: PPU, pp. 99-106. Disponible en:

$<<$ https://dialnet.unirioja.es/descarga/articulo/3411444.p df $>>$ [consultado el 15.09.2019].

HAMBROOK, Glyn. (2016). "Packaging a Posy of Perversity: Eduardo Marquina's 1905 Spanish Translation of Les Fleurs du mal". Bulletin of Hispanic Studies 93(9): 981-994.

LA ROCHEFOUCAULD, Edmée de. (1956). Anna de Noailles. París: Éditions Universitaires.

LAFARGA, Francisco. (2001). "Racine, P. de Olavide, R. Chacel: dos versiones de Phèdre a dos siglos de distancia". En: LAFARGA, Francisco \& DOMNÍNGUEZ, Antonio. (eds.). Los clásicos franceses en la España del siglo XX. Estudios de traducción y recepción. Barcelona: PPU, pp. 107-116. Disponible en:

$<<$ https://dialnet.unirioja.es/descarga/articulo/3411445.p df $>>$ [consultado el 15.09.2019].

---. (en prensa). "La poesía francesa en las antologías de Fernando Maristany". La Main de Thôt 7 (Défendre la traduction et l'interprétation).

LAMA, Miguel Ángel. (1999-2000). "Enrique Diez-Canedo y la poesía extranjera". Cauce 22-23: 191-228. Disponible en: 
“Transfer” XV: 1-2 (2020), pp. 351-370. ISSN: 1886-55

$<<$ http://institucional.us.es/revistas/cauce/22_23/art_10.p df $>>$ [consultado el 15.09.2019].

---. (2018). "Enrique Díez-Canedo en los cercados ajenos". En: LAFARGA, Francisco. (ed.). Creación y traducción en España (1898-1936): protagonistas de una historia. Kassel: Reichenberger, pp. 171-192.

LARNAC, Jean. (1931). Comtesse de Noailles. Sa vie, son œuvre. París, Éditions du Sagittaire.

LÉGLISE, Florence. (2014). "Marquina, premier traducteur des Fleurs du mal: face au rythme baudelairien, fine ou sourde oreille?". En: CARANDELL, Zoraida (ed.), Traduire pour l'oreille. Versions espagnoles de la prose et du théâtre poétiques français (1890-1930). París: Presses Sorbonne Nouvelle, pp. 115-130. Disponible en:

$<<\mathrm{https} / / /$ books.openedition.org/psn/570>> [consultado el 15.09.2019].

MARCO GARCÍA, Antonio. (2001). "Eduardo Marquina, traductor de Chénier y Prévost". En: LAFARGA, Francisco \& DOMÍNGUEZ, Antonio. (eds.) Los clásicos franceses en la España del siglo XX. Estudios de traducción y recepción. Barcelona: PPU, pp. 229-236. Disponible en:

$<<$ https://dialnet.unirioja.es/servlet/autor?codigo=93875>

$>$ [consultado el 15.09.2019].

MARTINEZ, Frédéric. (2018). Anna de Noailles. París: Gallimard.

MASSON, Georges-Armand. (1922). La comtesse de Noailles. Son ouvre. París: Éd. du Carnet Critique.

MIGNOT-OGLIASTRI, Claude. (1986). Anna de Noailles, une amie de la princesse Edmond de Polignac. París: MéridiensKlincksieck.

ORTEGA Y GASSET, José. (1923). "La poesía de Ana de Noailles". Revista de Occidente 1: 29-41, recogido también en: Obras completas. Madrid: Alianza-Revista de Occidente (1983), IV: 429-435.

PERRY, Catherine. (2003). Persephone Unbound. Dionysian Aesthetics in the Works of Anna de Noailles. Lewisburg, PA: Bucknell University Press. 
“Transfer” XV: 1-2 (2020), pp. 351-370. ISSN: 1886-55

PIQUER DESVAUX, Alicia. (1996). "Narcisismo, alteridad y vitalismo en la poesía de Anna de Noailles". En: SEGARRA, Marta \& CARABÍ, Àngels. (eds.), Amor e identidad. Barcelona: PPU, pp. 77-86. Disponible en:

$<<$ http://diposit.ub.edu/dspace/handle/2445/34250>> [consultado el 15.09.2019].

PUJANTE SEGURA, Carmen. (2014). De la novela corta y la "nouvelle" (1900-1950). Estudio comparativo entre escritoras. Madrid: Síntesis.

PYM, Anthony. (1995). "Translational and Non-Translational Regimes Informing Poetry Anthologies. Lessons on Authorship from Fernando Maristany and Enrique Díez-Canedo". En: KITTEL, Harald. (ed.), International Anthologies of Literature in Translation. Berlín: Eric Schmidt, pp. 251-270.

REMENTERÍA, Santiago. (2001). "Zuloaga, Noailles y Einstein: encuentros y alusiones". Bidebarrieta 9: 183-197. Disponible en:

$<<$ https://www.ehu.eus/ojs/index.php/Bidebarrieta/article /view/18427/0>> [consultado el 15.09.2019].

SÁEZ DELGADO, Antonio. (2007-2011). "Fernando Maristany y la traducción de poesía portuguesa en España a principios del siglo XX". Estudios Portugueses 7: 159-166.

VERONA, Roxana M. (2011). Parcours francophones: Anna de Noailles et sa famille culturelle. París: H. Champion.

ZARANDONA, Juan Miguel. (2017). "La visión fragmentaria de los versos de Alfred Tennyson traducidos al español en la antología de 1916 de Fernando Maristany". En: LAFARGA, Francisco. (ed.), La traducción fragmentaria: su lugar en antologías y revistas (1898-1936). Madrid: Guillermo Escolar, pp. 53-64.

Fecha de recepción: 10.10.2019 Fecha de aceptación: 21.10.2019 
“Transfer” XV: 1-2 (2020), pp. 351-370. ISSN: 1886-55

\begin{abstract}
Resumen:
Este trabajo, que pretende contribuir a un mejor conocimiento de la recepción española de escritoras francesas del rico periodo literario del fin de siglo y el primer tercio del siglo XX, se centra en la figura de Anna de Noailles (1876-1933, de soltera Anna Bibesco Bassarava de Brancovan). Tras poner de manifiesto el papel de gran figura social y literaria que jugó en la Francia de su tiempo, se pasa al estudio de su recepción en España, básicamente gracias a testimonios en la prensa de la época, y se insiste en particular en las traducciones de su obra, gran parte de ellas diseminadas en prensa y en antologías, aunque también en forma de libro, debidas principalmente a Tomás Borrás (1931), Rosa Chacel (1948) y Miriam Alonso Ribeiro (2011).
\end{abstract}

Palabras clave: Traducción; Recepción; Noailles, Anna de; siglo XX; España.

\title{
SPANISH TRANSLATIONS OF THE WORKS OF ANNA DE NOAILLES
}

Abstract:

This work, which aims to contribute to a better understanding of the Spanish reception of French women writers from the rich literary period of the end of the century and the first third of the 20th century, focuses on the figure of Anna de Noailles (18761933, born Anna Bibesco Bassarava de Brancovan). After revealing the role of a great social and literary figure that she played in the France of her time, we move on to the study of her reception in Spain, basically thanks to testimonies in the press of the time, and we insist in particular on the translations of her work, many of which are disseminated in the press and in anthologies, although also in book form, mainly due to Tomás Borrás (1931), Rosa Chacel (1948) and Mireia Alonso Ribeiro (2011).

Keywords: Translation; Reception; Noailles, Anna de; XXth Century; Spain. 